\title{
Determination of Critical Buckling Velocities of Pipes Conveying Fluid Rested on Different Supports Conditions
}

\author{
Muhsin J. Jweeg, PhD \\ Mechanical Engineering, \\ AL_Nahrain University \\ Baghdad,Iraq
}

\author{
Thaier J.Ntayeesh \\ Mechanical Engineering, \\ University of Baghdad \\ Baghdad,Iraq
}

\begin{abstract}
Pipes conveying fluid have significant applications in a wide range of industrial and engineering applications .Recently, this topic has been crystallized into a dynamical model for analyzing the fluid-structure-interaction (FSI) which has vast applications in aerospace ,aerodynamics, ship motion , medical engineering ..etc

The general solution for the vibration equation of conservative pipes conveying fluid is derived in this paper .From this solution the frequency and critical buckling velocity equations for pinned-pinned, clamped-pinned and clampedclamped pipes conveying fluid are obtained semi-analytically in terms of the pipe parameters.

A new experimental approach for estimating buckling critical velocities from measuring several natural frequencies at relatively small flow rates is presented. The results show good agreement between the estimated and theoretical critical velocities in case of pinned-pinned and clamped-pinned pipes .However for clamped-clamped pipes the accurate estimation requires higher flow rates. This method can serve using a relatively low pump discharge and simple fluid circuit instead of high discharge pumps which demand complication in the fluid circuits.
\end{abstract}

\section{Keywords}

Pipe conveying fluid, Critical buckling velocity, Stability

\section{INTRODUCTION}

Pipes conveying fluid are of considerable interest in many engineering fields. They are widely used in various industrial branches. Sometimes, their role is simply to transport fluids, as in oil pipelines, pump discharge lines, propellant lines and municipal water supply. In other cases, they provide basic structural components as in power plants, chemical plants, hydraulic systems, chemical plants, liquid-fuel rocket piping components, refrigerators, air-conditioners, heat exchangers, and so on.

The problem of pipes conveying fluid belongs to the broader class of dynamical problems involving axially moving continua such as traveling strings bands, belts, magnetic tapes and chain saws.

Due to its significance in science this topic may be taken as a new paradigm of interesting dynamical behavior to develop nonlinear dynamics, projecting them as a model dynamical problem[1,2].

In general ,the dynamical behavior of pipes conveying fluid are more complicated than the corresponding structure without fluid .In the latter case, free vibrations for a specified structure (degree of freedom and boundary conditions) depend only on its mass and stiffness .In such structures the Eigen values are related to structure parameters ,hence the natural frequency is unique .In this case the vibrations are of uncontrolled type .If however, such structures are subjected to an axial force, it is seen that the Eigen values are affected by the amount and direction of this force. In other words if this force is compressive the natural frequencies decrease with the increasing of this force. In this case vibration is controlled by this force and there is a critical value of this force at which the fundamental frequency drops to zero leading to buckling state.

When the structure interacts with a fluid media such as pipe conveying fluid different phenomena might occur according to the effects of the internal forces between the elastic structure and the fluid .Thus, the vibrations are of controlled type.

As compared with the ordinary elastic structures (without fluid), the linear dynamical behavior of pipe conveying fluid has the following significant differences[3];

1. It is complex boundary-value problems.

2. Classical normal (orthogonal) modes never exist).

3. The natural frequencies may become complex.

4. The natural frequencies are controlled by the fluidpipe parameters.

Housner[4] used an approximate power series for solving the governing equation but neglected the effect of internal pressure.

Noirdson[5] studied the stability for straight pipe simply supported with steady flow and he reached to the same conclusions which were made by the above authors, that the natural frequency for a system reduces with the increase in the fluid flow velocity, and the system losses stability by buckling.

Long [6] obtained the solution of the equation of motion using power series approximation. He studied the problem of fixedfree ended pipes as well for simply supported pipe.

Gregory and Paidoussis[7] and concluded, as did Benjamin, that the cantilever pipe loses its stability at a certain flow velocity by flutter rather than buckling.

Doare and Langre [8] studied instability of fluid conveying pipes on Winkler type foundation. The focus in their paper was on instability of infinitely long fluid conveying pipes using wave propagation approach, wherein results are interpreted in terms of static neutrality as criteria for pinnedpinned, clamped-clamped ends and dynamic neutrality for clamped-free ends. 


\section{DYNAMIC ANALYSIS OF PIPES CONVEYING FLUID}

Consider a straight uniform pipe conveying fluid of length $\mathrm{L}$. The following assumptions are considered in the analysis of the system under consideration [9]:

1. Neglecting the effect of gravity.

2. The pipe considered to be horizontal.

3 . Neglecting the material damping.

4. The pipe is inextensible.

5. Neglecting the shear deformation and rotary inertia.

6. All motion considered small.

7. Neglecting the velocity distribution through the crosssection of the pipe.

Derivation of the equation of motion for straight pipe with steady flow are available in the literature Ref.[5].For a singlespan pipe conveying fluid, the equation based on beam theory is given by,

$$
E I \frac{\partial^{4} y}{\partial x^{4}}+\left(M U^{2}+P A\right) \frac{\partial^{2} y}{\partial x^{2}}+2 M U \frac{\partial^{2} y}{\partial x \partial t}+(M+m) \frac{\partial^{2} y}{\partial t^{2}}=0
$$

where $F(x, t)$ : is the external harmonic force being applied normally to the pipe axis in the $y$-direction.

EI $\frac{\partial^{4} y}{\partial x^{4}}$ : Stiffness term

$\left(M U^{2}+P A\right) \frac{\partial^{2} y}{\partial x^{2}}$ : Curvature term

$2 M U \frac{\partial^{2} y}{\partial x \partial t}$ : Coriolis force term

$(M+m) \frac{\partial^{2} y}{\partial t^{2}}$ : Inertia force term

The Coriolis force is a result of the rotation of the system element due to the system lateral motion, since each point in the span rotates with angular velocity [10].

The equation of motion Eq.(1) can be written in the following non-dimensional form:

Where,

$$
\ddot{\eta}+2 M_{r} u_{0} \ddot{\eta}^{\prime}+\left[u_{0}^{2}+\Pi\right] \eta^{\prime \prime}+\eta^{(4)}=0
$$

$\xi=x / L$

$\eta=y / L$

$\eta^{(4)}=\frac{\partial^{4} y}{\partial x^{4}}, \eta^{(2)}=\frac{\partial^{2} y}{\partial x^{2}}, \ddot{\eta}^{\prime}=\frac{\partial^{2} y}{\partial x \partial t}, \ddot{\eta}=\frac{\partial^{2} y}{\partial t^{2}}$,

$\tau=\sqrt{E I /(M+M)}\left(\frac{t}{L^{2}}\right)$.

$\left.M_{r}=\sqrt{M /(M+m)}, u_{0}=U L \sqrt{(M / E I)} \quad{ }^{*}\right)$

$\Pi=\left(\frac{P A}{E I}\right) L^{2}$

$M_{r}$ : Non-dimensional mass ratio.

$\Pi$ : Non-dimensional fluid pressure.

The motion equation above is inhomogeneous, as the derivative coefficients of $\eta$ are explicit functions of $\xi$ and $\tau$. Then we discretize Eq. (2) using the Galerkin's method. Let $\eta(\xi, \tau)=\sum_{i=1}^{\infty} \phi_{i}(\xi) q_{i}(\tau)$

$q_{i}(\tau)$ is an generalized coordinate, $\phi_{i}(\xi)$ is an comparison function which satisfies all the boundary conditions. Selecting the first three orders conducts researches, which is:

$\eta(\xi, \tau)=\sum_{i=1}^{3} \phi_{i}(\xi) q_{i}(\tau)=\phi_{1}(\xi) q_{1}(\tau)+\phi_{2}(\xi) q_{2}(\tau)+$ $\phi_{3}(\xi) q_{3}(\tau)$

For pinned at both ends of pipes, its vibration model function is:

$\phi_{i}=\sqrt{2} \sin \left(\lambda_{i} \xi\right), i=1,2,3$

Where $\lambda 1, \lambda 2$ and $\lambda 3$ are beam eigenvalues $\lambda 1=\pi, \lambda 2=$ $2 \pi, \lambda 3=3 \pi$.
For fixed at both ends of pipes, its vibration model function is:

$$
\begin{aligned}
& \quad \Phi_{i}=\cosh \left(\lambda_{i} \xi\right)-\cos \left(\lambda_{i} \xi\right)+ \\
& \frac{\cosh \left(\lambda_{i}\right)-\cos \left(\lambda_{i}\right)}{\sinh \left(\lambda_{i}\right)-\sin \left(\lambda_{i}\right)}\left[\sin \left(\lambda_{i} \xi\right)-\sinh \left(\lambda_{i} \xi\right)\right], i=1,2,3
\end{aligned}
$$

Where $\lambda 1=4.7300, \lambda 2=7.8532, \lambda 3=10.9956$.

For fixed at one end and pinned at other end of pipes, its vibration model function is:

$\Phi_{i}=\cos \left(\lambda_{i} \xi\right)-\cosh \left(\lambda_{i} \xi\right)-$

$\frac{\cos \left(\lambda_{i}\right)-\cosh \left(\lambda_{i}\right)}{\sin \left(\lambda_{i}\right)-\sinh \left(\lambda_{i}\right)}\left[\sin \left(\lambda_{i} \xi\right)-\sinh \left(\lambda_{i} \xi\right)\right], i=1,2,3$

Where $\lambda 1=3.9267, \lambda 2=7.0686, \lambda 3=10.2102$

For cantilever pipe, its vibration model function is:

$\Phi_{i}=\cosh \left(\lambda_{i} \xi\right)-\cos \left(\lambda_{i} \xi\right)+$

$\frac{\sinh \left(\lambda_{i}\right)-\sin \left(\lambda_{i}\right)}{\cosh \left(\lambda_{i}\right)+\cos \left(\lambda_{i}\right)}\left[\sin \left(\lambda_{i} \xi\right)-\sinh \left(\lambda_{i} \xi\right)\right], i=1,2,3$

Where $\lambda 1=1.87512, \lambda 2=4.6941, \lambda 3=7.85476$

Eq.(3) is changed into matrix type, supposing $\Phi=$ $\left\{\begin{array}{l}\phi_{1} \\ \phi_{2} \\ \phi_{3}\end{array}\right\}, Q=\left\{\begin{array}{l}q_{1} \\ q_{2} \\ q_{3}\end{array}\right\}$, then

$$
\eta(\xi, \tau)=\Phi^{T} Q=Q^{T} \Phi
$$

Plugging (9) into (2), and supposing $H=u_{0}{ }^{2}+\Pi$, then:

$\phi^{T} \ddot{Q}+2 M_{r} u_{0} \phi^{T} \dot{Q}+H \phi^{\prime \prime} Q+\phi^{(4) T} Q=0$

By multiplying $\Phi=\left\{\begin{array}{l}\phi_{1} \\ \phi_{2} \\ \phi_{3}\end{array}\right\}$ with two sides of (10) and then

$\Phi \Phi^{T} \ddot{Q}+2 M_{r} u_{0} \Phi \Phi^{\prime T} \dot{Q}+H \Phi \Phi^{\prime T} Q+\Phi \Phi^{(4) T} Q$

Conducting $\xi$ integral to (11) at interval $[0,1]$, and substitutions based on orthogonality of trigonometric function:

$$
\begin{aligned}
& \int_{0}^{1} \phi \phi^{T} d \xi=I= \\
& \left(\begin{array}{ccc}
\int_{0}^{1} \phi_{1} \phi_{1}{ }^{T} & \int_{0}^{1} \phi_{2} \phi_{1}{ }^{T} & \int_{0}^{1} \phi_{3} \phi_{1}{ }^{T} \\
\int_{0}^{1} \phi_{1} \phi_{2}{ }^{T} & \int_{0}^{1} \phi_{2} \phi_{2}{ }^{T} & \int_{0}^{1} \phi_{3} \phi_{2}{ }^{T} \\
\int_{0}^{1} \phi_{1} \phi_{3}{ }^{T} & \int_{0}^{1} \phi_{2} \phi_{3}{ }^{T} & \int_{0}^{1} \phi_{3} \phi_{3}{ }^{T}
\end{array}\right) d \xi= \\
& \left(\begin{array}{lll}
1 & & \\
& 1 & \\
& & 1
\end{array}\right) \text {, } \\
& \int_{0}^{1} \phi \phi^{\prime T} d \xi=B= \\
& \left(\begin{array}{ccc}
\int_{0}^{1} \phi_{1} \phi_{1}{ }^{\prime} T & \int_{0}^{1} \phi_{2} \phi_{1}{ }^{\prime} T & \int_{0}^{1} \phi_{3} \phi_{1}{ }^{\prime} T \\
\int_{0}^{1} \phi_{1} \phi_{2}{ }^{\prime} T & \int_{0}^{1} \phi_{2} \phi_{2}{ }^{\prime} T & \int_{0}^{1} \phi_{3} \phi_{2}{ }^{\prime} T \\
\int_{0}^{1} \phi_{1} \phi_{3}{ }^{\prime} T & \int_{0}^{1} \phi_{2} \phi_{3}{ }^{\prime T} & \int_{0}^{1} \phi_{3} \phi_{3}{ }^{\prime} T
\end{array}\right) d \xi= \\
& \left(\begin{array}{lll}
b_{11} & b_{12} & b_{13} \\
b_{21} & b_{22} & b_{23} \\
b_{31} & b_{32} & b_{33}
\end{array}\right), \int_{0}^{1} \phi \phi^{\prime \prime T} d \xi=C= \\
& \left(\begin{array}{lll}
\int_{0}^{1} \phi_{1} \phi_{1}{ }^{\prime \prime} \mathrm{T} & \int_{0}^{1} \phi_{2} \phi_{1}{ }^{\prime \prime} \mathrm{T} & \int_{0}^{1} \phi_{3} \phi_{1}{ }^{\prime \prime} \mathrm{T} \\
\int_{0}^{1} \phi_{1} \phi_{2}{ }^{\prime \prime} \mathrm{T} & \int_{0}^{1} \phi_{2} \phi_{2}{ }^{\prime \prime} \mathrm{T} & \int_{0}^{1} \phi_{3} \phi_{2}{ }^{\prime \prime} \mathrm{T} \\
\int_{0}^{1} \phi_{1} \phi_{3}{ }^{\prime \prime} \mathrm{T} & \int_{0}^{1} \phi_{2} \phi_{3}{ }^{\prime \prime} \mathrm{T} & \int_{0}^{1} \phi_{3} \phi_{3}{ }^{\prime \prime} \mathrm{T}
\end{array}\right) \mathrm{d} \xi= \\
& \left(\begin{array}{lll}
\mathrm{c}_{11} & \mathrm{c}_{12} & \mathrm{c}_{13} \\
\mathrm{c}_{21} & \mathrm{c}_{22} & \mathrm{c}_{23} \\
\mathrm{c}_{31} & \mathrm{c}_{32} & \mathrm{c}_{33}
\end{array}\right) \int_{0}^{1} \phi \phi^{(4) T} d \xi=\Lambda=
\end{aligned}
$$




$$
\begin{aligned}
& \left(\begin{array}{lll}
\int_{0}^{1} \phi_{1} \phi_{1}{ }^{(4) T} & \int_{0}^{1} \phi_{2} \phi_{1}{ }^{(4) T} & \int_{0}^{1} \phi_{3} \phi_{1}{ }^{(4) T} \\
\int_{0}^{1} \phi_{1} \phi_{2}{ }^{(4) T} & \int_{0}^{1} \phi_{2} \phi_{2}{ }^{(4) T} & \int_{0}^{1} \phi_{3} \phi_{2}{ }^{(4) T} \\
\int_{0}^{1} \phi_{1} \phi_{3}{ }^{(4) T} & \int_{0}^{1} \phi_{2} \phi_{3}{ }^{(4) T} & \int_{0}^{1} \phi_{3} \phi_{3}{ }^{(4) T}
\end{array}\right) d \xi= \\
& \left(\begin{array}{ccc}
\lambda_{1}{ }^{4} & & \\
& \lambda_{2}{ }^{4} & \\
& & \lambda_{3}{ }^{4}
\end{array}\right)
\end{aligned}
$$

$\phi_{1}, \phi_{2}$ and $\phi_{3}$ are the first three mode functions for specific boundary conditions.

For pinned at both ends of pipes, the matrix $\mathrm{B}$ and $\mathrm{C}$ are:

$$
\begin{aligned}
B=\left(\begin{array}{ccc}
0 & -2.6667 & 0 \\
2.6667 & 0 & -4.8 \\
0 & 4.8 & 0
\end{array}\right), C & \\
& =\left(\begin{array}{ccc}
-\left(\pi^{2}\right) & 0 & 0 \\
0 & -\left(2 \pi^{2}\right) & 0 \\
0 & 0 & -\left(3 \pi^{2}\right)
\end{array}\right)
\end{aligned}
$$

For fixed at both ends of pipes, the matrix $\mathrm{B}$ and $\mathrm{C}$ are:

$$
\begin{aligned}
B=\left(\begin{array}{ccc}
0 & -3.3421 & 0 \\
3.3421 & 0 & -5.5161 \\
0 & 5.5161 & 0
\end{array}\right), C & \\
& =\left(\begin{array}{ccc}
-12.3028 & 0 & 9.7315 \\
0 & -46.0501 & 0 \\
9.7315 & 0 & -98.9047
\end{array}\right)
\end{aligned}
$$

For fixed at one end and pinned at other end of pipes, the matrix $\mathrm{B}$ and $\mathrm{C}$ are:

$$
\begin{aligned}
& B=\left(\begin{array}{ccc}
0 & -2.9965 & 0.3167 \\
2.9965 & 0 & -5.1468 \\
-0.3167 & 5.1468 & 0
\end{array}\right), C \\
&=\left(\begin{array}{ccc}
-11.5126 & 4.2814 & 3.7993 \\
4.2814 & -42.8964 & 7.81913 \\
3.7993 & 7.8191 & -94.0376
\end{array}\right)
\end{aligned}
$$

For cantilever pipe, the matrix $\mathrm{B}$ and $\mathrm{C}$ are:

$$
\begin{aligned}
B=\left(\begin{array}{ccc}
2 & -4.75948 & 3.78433 \\
0.75948 & 2 & -6.22218 \\
0.21566 & 2.22218 & 2
\end{array}\right), C & \\
& =\left(\begin{array}{ccc}
0.8581 & -11.7433 & 27.4531 \\
1.8738 & -13.2942 & -9.04205 \\
1.56453 & 3.22935 & -45.9043
\end{array}\right)
\end{aligned}
$$

Using equations of (12), the discretized equation after reduced order through (11) is showed below:

$I \ddot{Q}+2 M_{r} u_{0} B \dot{Q}+(C H+\Lambda) Q=0$

Where $\ddot{Q}=\left\{\begin{array}{l}\ddot{q}_{1} \\ \ddot{q}_{2} \\ \ddot{q}_{3}\end{array}\right\}, \dot{Q}=\left\{\begin{array}{l}q_{1} \\ q_{2} \\ q_{3}\end{array}\right\}, Q=\left\{\begin{array}{l}q_{1} \\ q_{2} \\ q_{3}\end{array}\right\}$
When $\dot{Q}=\Omega i, \ddot{Q}=-\Omega^{2}$, and Eq. (13) become;

$$
\begin{aligned}
& {\left[-I \Omega^{2}+2 M_{r} u_{0} B \Omega i+(C H+\Lambda)\right] Q=0} \\
& {\left[-I \Omega^{2}+2 M_{r} u_{0} B \Omega i+(C H+\Lambda)\right]=S=} \\
& \left(\begin{array}{lll}
\mathrm{s}_{11} & \mathrm{~s}_{12} & \mathrm{~s}_{13} \\
\mathrm{~s}_{21} & \mathrm{~s}_{22} & \mathrm{~s}_{23} \\
\mathrm{~s}_{31} & \mathrm{~s}_{32} & \mathrm{~s}_{33}
\end{array}\right) \\
& \text { Where } \\
& \qquad \mathrm{s}_{11}=\lambda_{1}{ }^{4}+H \mathrm{c}_{11}+2 M_{r} u_{0} \mathrm{~b}_{11} \Omega i-\Omega^{2} \\
& \mathrm{~s}_{12}=H \mathrm{c}_{12}+2 M_{r} u_{0} \mathrm{~b}_{12} \Omega i \\
& \mathrm{~s}_{13}=H \mathrm{c}_{13}+2 M_{r} u_{0} \mathrm{~b}_{13} \Omega i \\
& \mathrm{~s}_{21}=H \mathrm{c}_{21}+2 M_{r} u_{0} \mathrm{~b}_{21} \Omega i \\
& \mathrm{~s}_{22}=\lambda_{2}{ }^{4}+H \mathrm{c}_{22}+2 M_{r} u_{0} \mathrm{~b}_{22} \Omega i-\Omega^{2} \\
& \mathrm{~s}_{23}=H \mathrm{c}_{23}+2 M_{r} u_{0} \mathrm{~b}_{23} \Omega i \\
& \mathrm{~s}_{31}=H \mathrm{c}_{31}+2 M_{r} u_{0} \mathrm{~b}_{31} \Omega i \\
& \mathrm{~s}_{31}=H \mathrm{c}_{31}+2 M_{r} u_{0} \mathrm{~b}_{31} \Omega i \\
& \mathrm{~s}_{32}=H \mathrm{c}_{32}+2 M_{r} u_{0} \mathrm{~b}_{32} \Omega i \\
& \mathrm{~s}_{33}=\lambda_{3}{ }^{4}+H \mathrm{c}_{33}+2 M_{r} u_{0} \mathrm{~b}_{33} \Omega i-\Omega^{2}
\end{aligned}
$$

The natural frequency $(\Omega)$ is evaluated by setting $|S|$ equal to zero. Expansion of this determent leads to the following characteristic equation;

$\Omega^{6}-k_{5} \Omega^{5} i-k_{4} \Omega^{4}-k_{3} \Omega^{3} i-k_{2} \Omega^{2}-k_{1} \Omega i-k_{0}=0$ (15) Where $k_{5}, k_{4}, k_{3}, k_{2}, k_{1}$ and $k_{0}$ are constants depending on the boundary conditions as shown in appendix Table (1)

\section{EXPERIMENTAL WORK}

The aim of this work is to measure the natural frequencies of PVC pipes conveying water at different boundary conditions, study the effect of fluid velocity on the natural frequencies and estimation of critical buckling velocities .

\subsection{Test Models.}

For measuring the natural frequencies and investigating the effect of the fluid velocity on the natural frequencies, PVC pipe model was prepared. Table (2) shows the main specifications of this model.

Table ( 2): Specifications of the test models

\begin{tabular}{|l|l|l|l|l|l|}
\hline $\begin{array}{l}\text { Model } \\
\text { No. }\end{array}$ & Material & $\mathbf{D}_{\mathbf{o}}(\mathbf{m})$ & $\begin{array}{l}\text { Thick. } \\
(\mathbf{m})\end{array}$ & $\begin{array}{l}\boldsymbol{\rho} \\
\mathbf{k g} / \mathbf{m} 3\end{array}$ & $\begin{array}{l}\mathbf{E} \\
\mathbf{G N} / \mathbf{m}^{\mathbf{2}}\end{array}$ \\
\hline 1 & PVC & 0.020 & 0.0018 & 975 & 6.83 \\
\hline
\end{tabular}

\subsection{Experimental Rig}

The rig consists of two main parts; the foundation and the two Substrates. It was constructed from $(120 \mathrm{~cm} \times 40 \mathrm{~cm} \times 1 \mathrm{~cm})$ rectangular section iron plate foundation and two $(22 \mathrm{~cm} \times 20 \mathrm{~cm} \times 1 \mathrm{~cm})$ Substrates of pipe support as shown in appendix [Fig.(1)]

The two Substrates were designed to fulfill the various requirements for pinned and clamped conditions. They consisted of two main parts; the iron base and the ball bearing case. The ball bearing has free vertical movement, which leads to zero moment in pin support. 
Table(2): The Parameters Constants of Eq.(15)

\begin{tabular}{|c|c|c|c|}
\hline & Pinned-Pinned & Clamped-Clamped & Clamped-Pinned \\
\hline $\boldsymbol{k}_{5}$ & $\mathbf{0}$ & $\mathbf{0}$ & $\mathbf{0}$ \\
\hline $\boldsymbol{k}_{4}$ & $\begin{array}{c}-138.175 H \\
+9546.1 \\
+120.608 r^{2} u_{o}^{2}\end{array}$ & $\begin{array}{c}-157.258 H \\
+18922 \\
+166.388 r^{2} u_{o}^{2}\end{array}$ & $\begin{array}{c}-148.447 \mathrm{H} \\
+13601.8 \\
+142.275 \mathrm{mr}^{2} u_{o}{ }^{2}\end{array}$ \\
\hline$k_{3}$ & $\mathbf{0}$ & 0 & $\begin{array}{l}+0.003 T m r u_{o} \\
-0.001 m r^{3} u_{o}{ }^{3}\end{array}$ \\
\hline $\boldsymbol{k}_{2}$ & $\begin{array}{c}+3436.55 m r^{2} u_{o}{ }^{2} H \\
-233439 m r^{2} u_{o}^{2} \\
-4773.04 H^{2} \\
555683 H \\
-0.132176 * 10^{8}\end{array}$ & $\begin{array}{c}+4481.05 m r^{2} u_{o}{ }^{2} H \\
-714029 m r^{2} u_{o}{ }^{2} \\
-6243.22 H^{2} \\
0.134854 * 10^{7} H \\
-0.648214 * 10^{8}\end{array}$ & $\begin{array}{c}+4030.57 m r^{2} u_{o}{ }^{2} H \\
-416514 m r^{2} u_{o}{ }^{2} \\
-5516.43 H^{2} \\
887358 H \\
-0.303083 * 10^{8}\end{array}$ \\
\hline $\boldsymbol{k}_{1}$ & 0 & 0 & $\begin{array}{l}-0.0613238 T m r u_{o} \\
\quad+0.005 T^{2} m r u_{o}\end{array}$ \\
\hline$k_{0}$ & $\begin{array}{c}-34609.9 H^{3} \\
+0.478222 * 10^{7} H^{2} \\
-0.165195 * 10^{9} H \\
+0.119786 * 10^{10}\end{array}$ & $\begin{array}{c}-51673 H^{3} \\
+0.148296 * 10^{8} H^{2} \\
-0.120933 * 10^{10} H \\
+0.278330 * 10^{11}\end{array}$ & $\begin{array}{c}-43139.2 H^{3} \\
+0.877896 * 10^{7} H^{2} \\
-0.478995 * 10^{9} H \\
+0.645029 * 10^{10}\end{array}$ \\
\hline
\end{tabular}

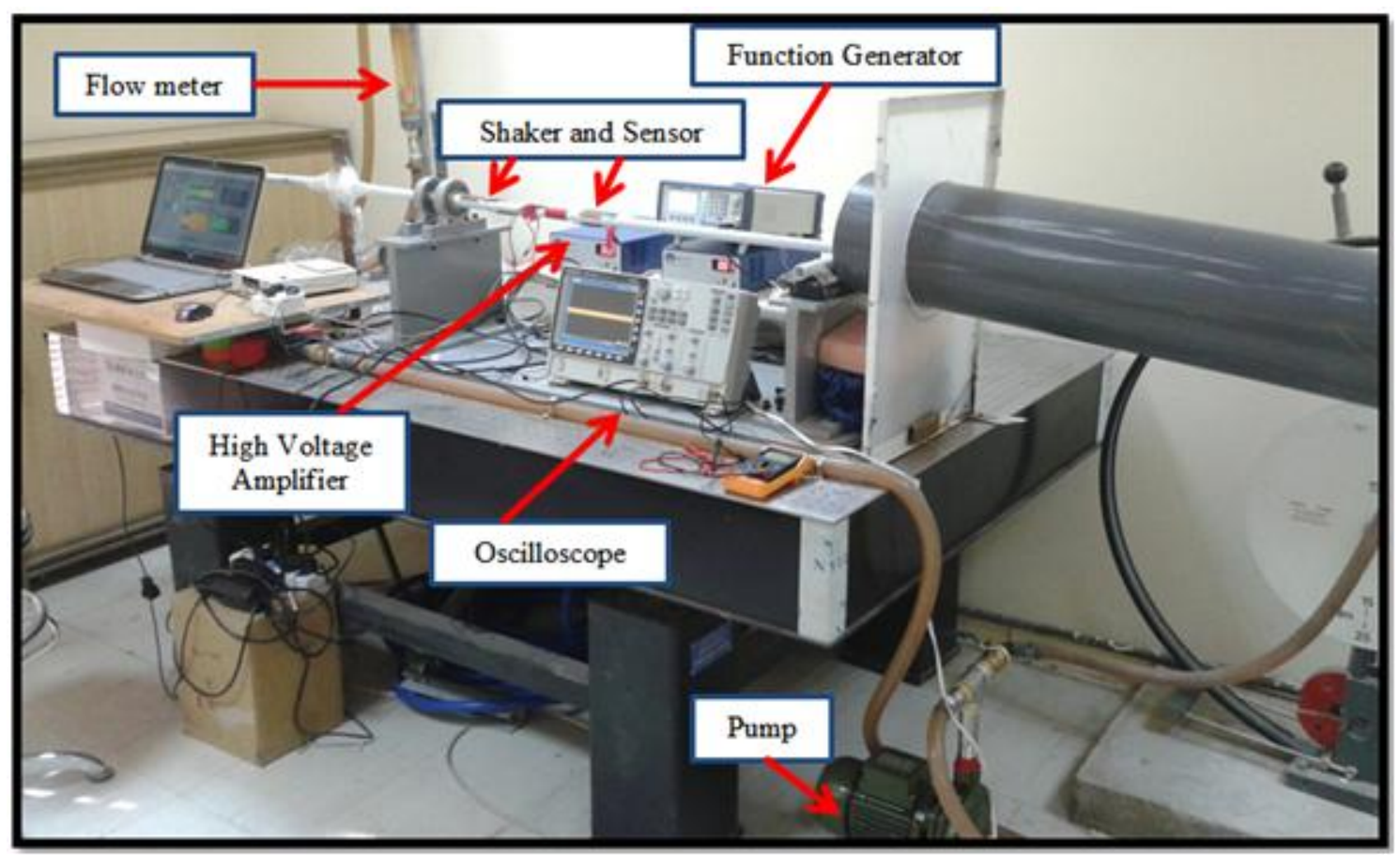

Fig.(1): Modal structure of pipe conveying fluid

\subsection{Water Circuit.}

In all tests, water was used as a flowing fluid .To measure the water flow rate a flowmeter was fitted at the inlet of the test model. The water circuit is shown in Fig.(3). The main components of this circuit are the collecting tank (150 Lit.), centrifugal pump (100 Lit/min., $25 \mathrm{~m}$ ), control valve (gate type) and the test pipe model. 


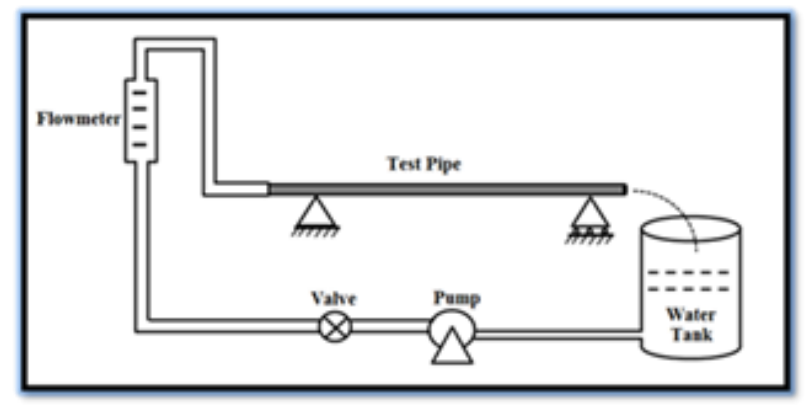

Fig.(3): Schematic diagram of water circuit and the test model

\subsection{Instruments and Test Procedure.}

The block diagram of the various components of the electric vibration instrument are shown in Fig.(4).

The electrical vibration circuit can be divided into two main circuits; the first represents the "excitation side" which consists of: function generator, high voltage power amplifier and vibration exciter ( piezoelectric shaker). The second is the "response side" which includes: piezoelectric sensor and oscilloscope, as shown in Fig.(1) . A photograph of the electrical instruments is shown in Fig.(4).

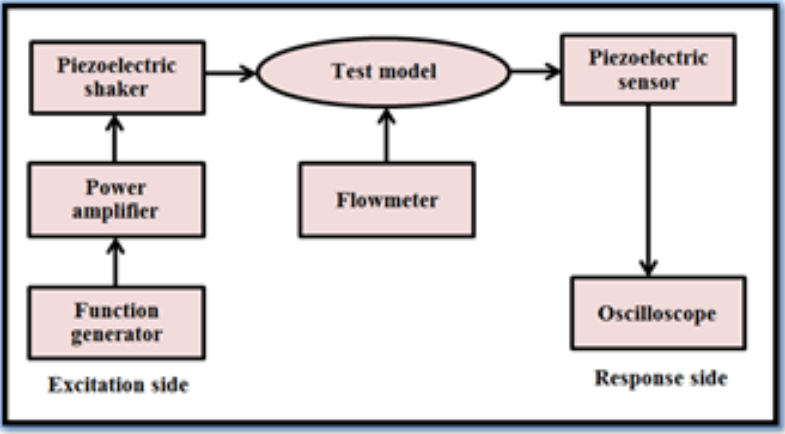

Fig.(4): Block diagram of the measuring circuit

\subsubsection{High Voltage Amplifier of PZT (HVPZT).}

A power amplifier drives piezoelectric actuators through an applied voltage. The device used in the series of experiments featured in this work is a (Trek Model 2205) high voltage inverted operational amplifier

\subsubsection{Sensor and Shaker}

QUICKPACK MIDE QP20W double layer piezoelectric type is use as structural sensors transducers and shaker

\subsubsection{Function Generator}

BK model 4075, a $25 \mathrm{MHz}$ arbitrary/Function generator, is a precision source of sine, triangle, square and pulse waveforms plus dc voltage. All can be externally modulated. Output can be continuous or can be triggered or gated by external signal or front panel switch. Amplitude of the waveforms is variable from $30 \mathrm{~V}$ down to $1.5 \mathrm{mV}$. DC reference of the waveform can be offset positively or negatively.

\subsubsection{Oscilloscope}

The digital oscilloscope $250 \mathrm{MHz}$ type GW-INSTEK GDS3000 is used with built-in FFT analyzer. This device is used to display the vibration waves and frequency results, which extract from the sensor, due to vibration of the structures.

\section{RESULTS AND DISCUSSION \\ 4.1 Buckling Critical Velocities of Conservative Pipes}

The critical velocities initiating buckling for the three conservative pipes are shown in Figs.(5 to 7 ) .In plotting these figures the general equations were used when the natural frequency become zero value .

At zero pressure $(\Pi=0)$, Figs. (5 to 7 ) give the following values of critical velocities: $\pi, 1.43 \pi$ and $2 \pi$ for pinnedpinned, clamped-pinned and clamped-clamped pipes, respectively.These is identical to the Euler load of buckling for the corresponding beams with compressive axial force.

As the pressure increases, these figures show that the critical velocities decrease linearly. This is due to the effect of increasing the compressive force exerted on pipe ends as the pressure increases.

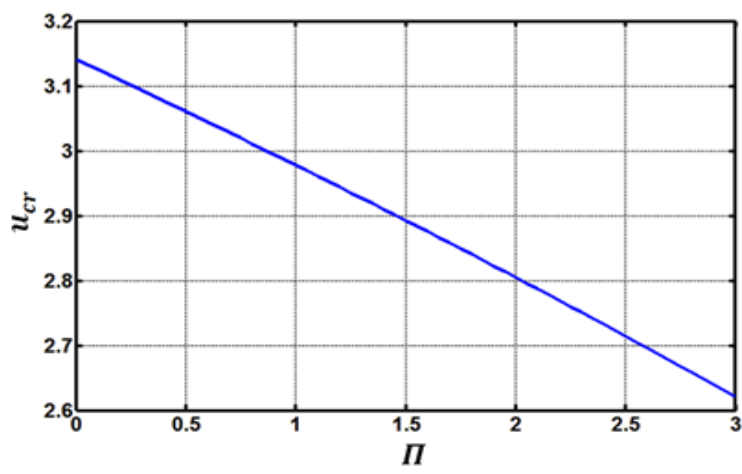

Fig.(5): Critical velocities of buckling at different pressures of $\mathbf{p}-\mathbf{p}$ pipes

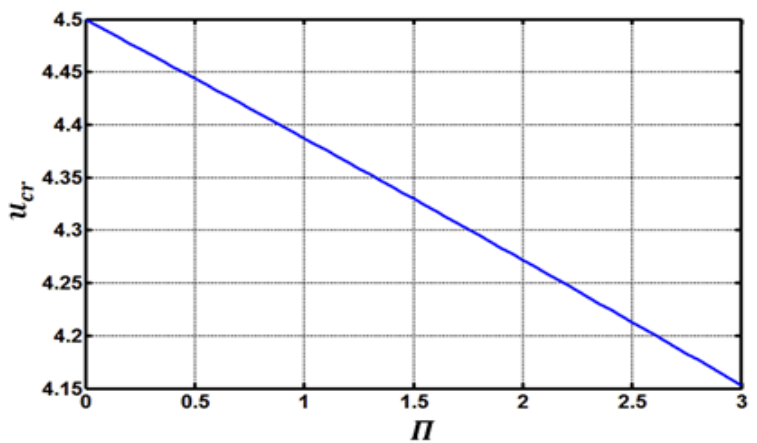

Fig.(6): Critical velocities of buckling at different pressure of c-p pipes

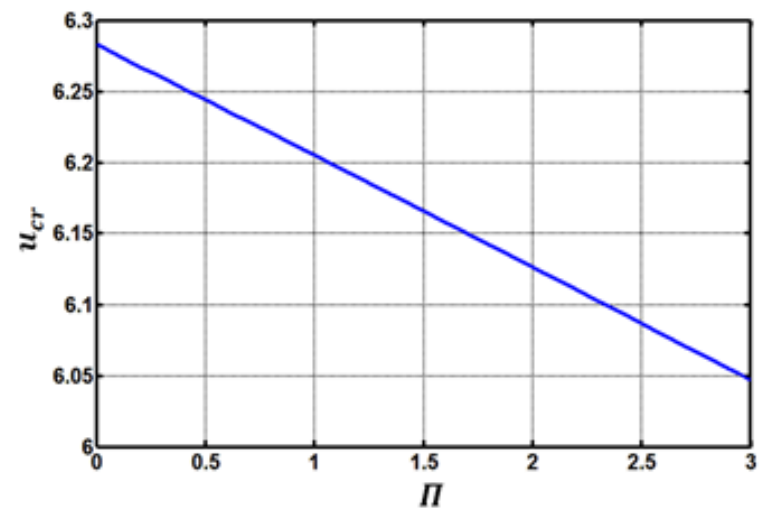

Fig.(7):Critical velocities of buckling at different pressure of c-c pipes 


\subsection{Stability Boundary}

For the purpose of illustration of this approach, a typical plot of the stability boundary for clamped-pinned pipes conveying fluid at $\Pi=0$ and $M_{r}=0.9$ is constructed in Fig.(8).To construct such a figure the roots of general equation are evaluated for various values of the dimensionless velocity $u_{0}$. It should be noticed that the sixth order polynomial equation like Eq. (15) gives six roots for $\Omega$.However in this case each two of them are equal but with opposite signs .If these roots are squared then only three values of $\Omega^{2}$ are different . Finally the first two values of $\Omega^{2}$ and $u_{0}{ }^{2}$ are plotted to get the root locus of two modes as shown in this figure.

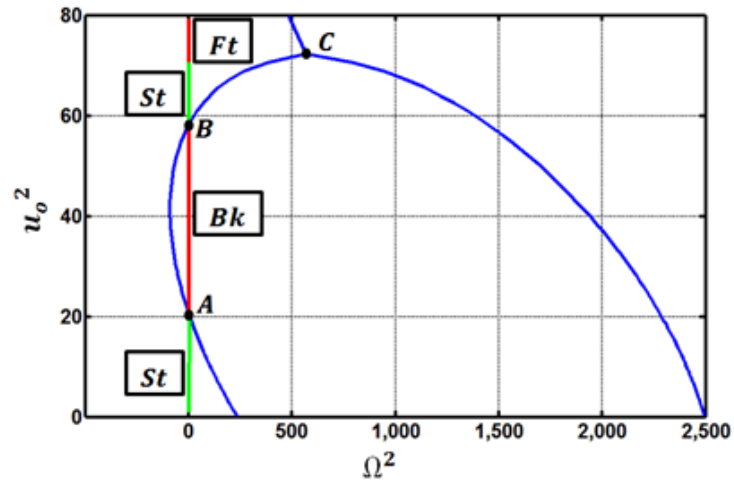

Fig.(8):Stability boundary of c-p pipes at $\Pi=0, M_{r}=$ 0.5 (St: Stable, Bk: buckling, Ft: flutter)

To inspect the stability behavior the following rules are followed:

1. When all the roots lie in the first quarter (or to the right of the line $\Omega^{2}=0$ ) in $\Omega^{2}-u_{0}^{2}$ plane ,the pipe is stable .

2. If at least one of the two roots lies in the second quarter, the pipe is unstable.

3. Buckling instability initiates at the points of intersection of the root locus with the line $\Omega^{2}=0$.

4. Flutter instability initiates at the maximum point of the root locus[11].

Now, referring to Fig. (8) the following sequence of the stability behaviors can be observed:-

- At $u_{0}^{2} \in\left[\begin{array}{ll}0 & 20\end{array}\right]$, the pipe is stable since all values of $\Omega^{2}$ lie to the right of the line $\Omega^{2}=0$.

- At $u_{0}^{2} \in\left[\begin{array}{ll}20 & 59\end{array}\right]$, the pipe is under buckling instability since some values of $\Omega^{2}$ lie to the left.

- At $u_{0}^{2} \in\left[\begin{array}{ll}59 & 72\end{array}\right]$, the pipe regains its stability as in the first case.

- For $u_{0}^{2}>72$ the pipe is at flutter instability.

- Points A and B are the critical points of buckling instability since they lie on the line $\Omega^{2}=0$.

- Point $\mathrm{C}$ is the critical point of flutter instability which is the maximum point in the plot.

The stability boundaries for pinned-pinned, clamped-pinned and clamped-clamped pipes are shown in Figs.(9 to 11) .To investigate the effect of the mass ratio $M_{r}$ on stability of these three pipes, plots for $M_{r}=0.3,0.5$ and 0.9 are presented in these figures .As it is clear from these figures ,the effect of varying $M_{r}$ is so significant on flutter instability since the maximum points on the plots are either shifted to the right as $M_{r}$ increased or it may vanish as in Fig.(10) where $M_{r}=0.3$. It should be noted here that, according to this effect the sequence of stability is noticeably altered. For example in Fig. (9) at $M_{r}=0.3$ the sequence of stability is: - stable, buckling, and flutter while at $M_{r}=0.9$ it becomes: - stable, buckling, stable and flutter. This is also true for the other figures .However $M_{r}$ has no effect on buckling instability since the critical points of buckling (the intersection points with line $\Omega^{2}=0$ ) are not changed for all values of $M_{r}$.

To check the validity of the presented approach selected points from Figs.(9to 11) will be compared with the available results in the literature .For example at $u_{0}^{2}=0$ ,Figs.(9 to 11) give the squarer of the first and second natural frequencies of corresponding beams which are 97.4 and 1558.54 for pinned-pinned, 237.74 and 2496.51 for clampedpinned and 500.6 and 3803.14 for clamped-clamped pipes[90]. This is true since in this case, the pipe is reduced to a beam as the fluid velocity becomes zero. Also, in Figs.(9 to 11) the lowest points of intersections of the plots with the line $\Omega^{2}=0$ for any $M_{r}$ are $9.61,20.25$ and 40.7 ,respectively .These are nearly the square of $\pi, 1.43 \pi$ and $2 \pi$ respectively ,which are the critical velocities for first mode buckling of these pipes.

The fundamental natural frequencies $\Omega_{1}$ at $\Pi=0$ of the three conservative pipes can be calculated from the following approximated formulas[12]:-

$\Omega_{1}=\pi^{2} \sqrt{1-\frac{u_{o}^{2}}{\pi^{2}}}$

$\Omega_{1}=3.93^{2} \sqrt{1-0.747 \frac{u_{o}^{2}}{\pi^{2}}}$

$\Omega_{1}=4.73^{2} \sqrt{1-0.55 \frac{u_{o}^{2}}{\pi^{2}}}$

Eq.(15) can be used to calculate $\Omega^{2}$ at pre-critical fluid velocities. By simple calculations it is easy to verify that the results of Eq. 15) coincide with those presented in Figs. (9 to 11).

The effect of the fluid pressure is studied in Figs.(12 to 14) where $M_{r}=0$ and $\Pi=0,2$ and 3 were chosen .It is clear from these figures that the effect of increasing $\Pi$ is to slightly shift the boundary of stability to lower values for all of the considered pipes .However the sequences of stability are not altered .

It is of interesting to state that; the critical velocities of buckling of the three conservative pipes can be evaluated directly from the Eq.(15) of the root locus by considering the following analysis:-

The general form of root locus is:-

$\Omega^{6}-k_{5} \Omega^{5} i-k_{4} \Omega^{4}-k_{3} \Omega^{3} i-k_{2} \Omega^{2}-k_{1} \Omega i-k_{0}=0$ (15)

Buckling initiates at $\Omega=0$, so that Eq.(15) gives the following condition for buckling instability :-

$k_{0}=0$

Appling this condition to Table (2-1), one gets the following equations;

$-34609.9 H^{3}+0.478222 * 10^{7} H^{2}-0.165195 * 10^{9} H+$

$0.119786 * 10^{10}=0 \quad(\mathrm{p}-\mathrm{p})$

$-43139.2 H^{3}+0.877896 * 10^{7} H^{2}-0.478995 * 10^{9} H+$

$0.645029 * 10^{10}=0 \quad(\mathrm{c}-\mathrm{p})$

$-51673 H^{3}+0.148296 * 10^{8} H^{2}-0.120933 * 10^{10} H+$

$0.278330 * 10^{11}=0 \quad(\mathrm{c}-\mathrm{c})$

where, $H=u_{0}^{2}+\Pi$

From Eqs.(18) the critical velocities of buckling can be evaluated for the three types of conservative pipes at a given value of $\Pi$. It is clear from these equations that the critical velocities are independent of the mass ratio $M_{r}$.

Flutter instability initiates when the natural frequency become complex with negative imaginary parts and represent the maximum point in the plot of stability. 
The values of the critical velocities of buckling and flutter, for the three pipes at $\Pi=0$ and $M_{r}=0.9$ are presented in Table (6-2).

Table (3) indicates that the critical velocities coincide with those shown in Figs. ( 9 to 11 ) at $M_{r}=0.9$.

Table(3): Buckling and flutter critical velocities of conservative pipes $\Pi=0$ and $M_{r}=0.9$.

\begin{tabular}{|l|l|l|l|}
\hline $\begin{array}{l}\text { Boundary } \\
\text { conditions }\end{array}$ & $\begin{array}{l}1^{\text {st }} \begin{array}{c}u_{0} \\
\text { Buckling }\end{array} \\
\text { B-p }\end{array}$ & $\begin{array}{l}2^{\text {nd }} u_{0} \\
\text { Buckling }\end{array}$ & $\begin{array}{c}u_{0} \\
\text { Flutter }\end{array}$ \\
\hline c-p & $1.43 \pi$ & $2 \pi$ & 6.7 \\
\hline c-c & $2 \pi$ & $2.5 \pi$ & 8.5 \\
\hline
\end{tabular}

Table (6-2) indicates that conservative pipes can lose their stability by buckling prior to flutter as the fluid velocity increase.

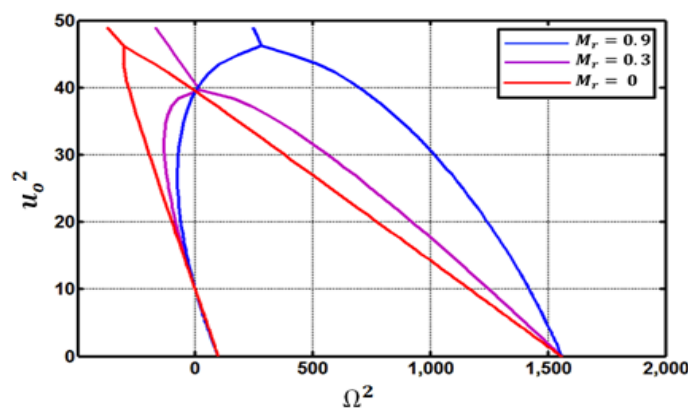

Fig.(9): Stability boundary of p-p pipe at $\Pi=0$.

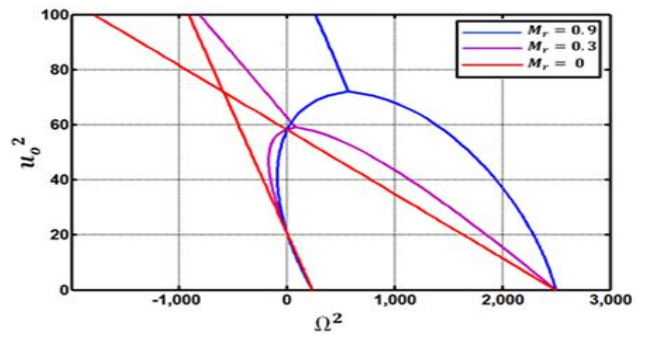

Fig.(10): Stability boundary of c-p pipe at $\Pi=0$.

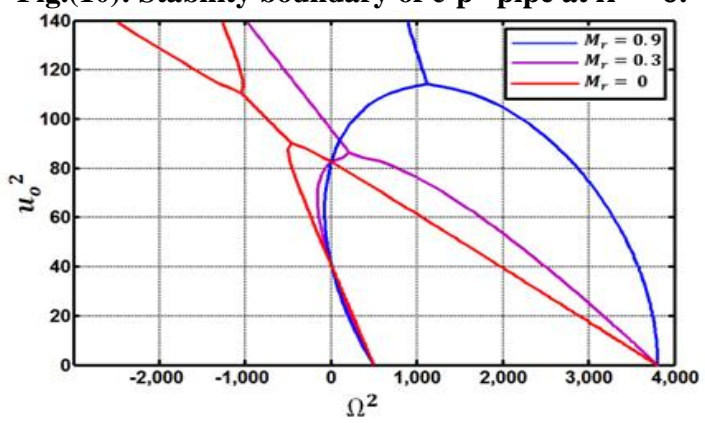

Fig.(11): Stability boundary of c-c pipe at $\Pi=0$.

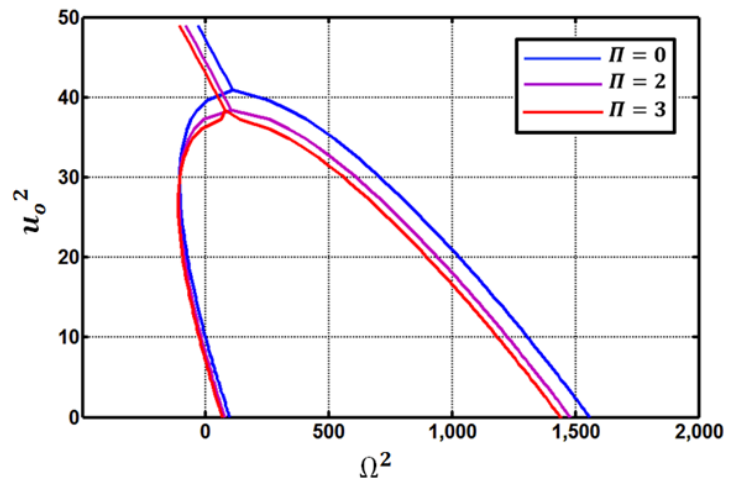

Fig.(12): Stability boundary of p-p pipe at $M_{r}=0$.

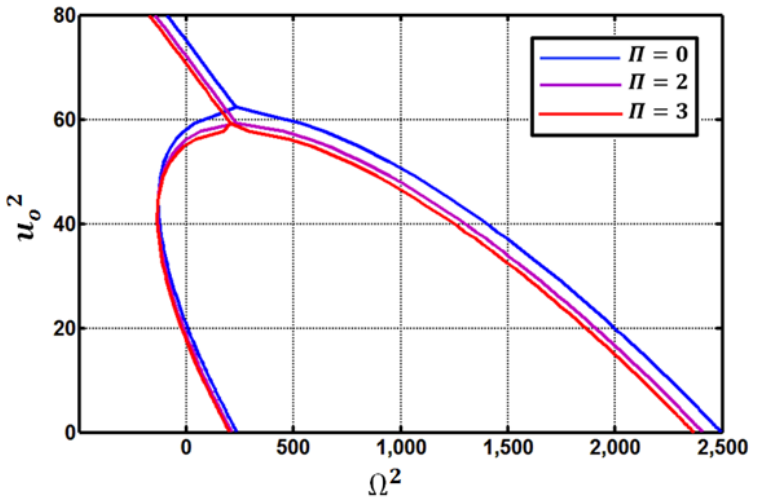

Fig.(13): Stability boundary of c-p pipe at $M_{r}=0$.

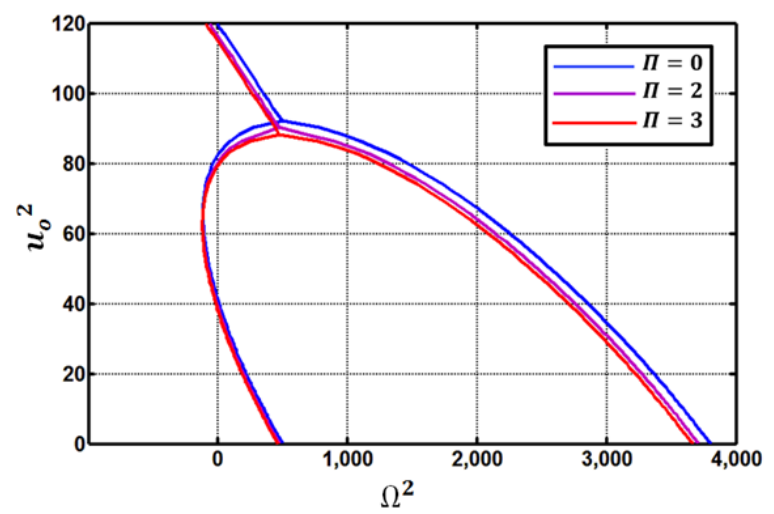

Fig.(14): Stability boundary of c-c pipe at $M_{r}=0$.

\subsection{Estimating of the Critical Velocities}

The measured fundamental natural frequencies and the associated fluid flow rates for PVC pipe model, at different boundary conditions are given in Table (4) .

Table( 4): Measured fundamental natural frequencies (Hz) for $1 \mathrm{~cm}$ diameter PVC pipe at different boundary conditions

\begin{tabular}{|l|l|l|l|l|l|l|l|}
\hline $\mathrm{Q}(1 / \mathrm{min})$ & 0 & 10 & 20 & 30 & 40 & 50 & 60 \\
\hline p-p & 7.2 & 6.6 & 6 & 5.9 & 5.4 & 5 & 2.5 \\
\hline c-p & 8.1 & 8.2 & 8 & 7.75 & 7.3 & 6.7 & 6 \\
\hline c-c & 12 & 11.8 & 11.5 & 11.2 & 11 & 10.4 & 9.9 \\
\hline
\end{tabular}

The dimensionless frequencies $\Omega$ and velocities $U$ of the measured data were calculated from Eqs.(15 and 18). 
In Eigs (15 to 17) the values of $\Omega^{2}$ and $U^{2}$ for model and at the three considered boundary conditions are plotted and fitted to straight lines. Then, the equations of the fitted lines were evaluated and are given in Table (5).

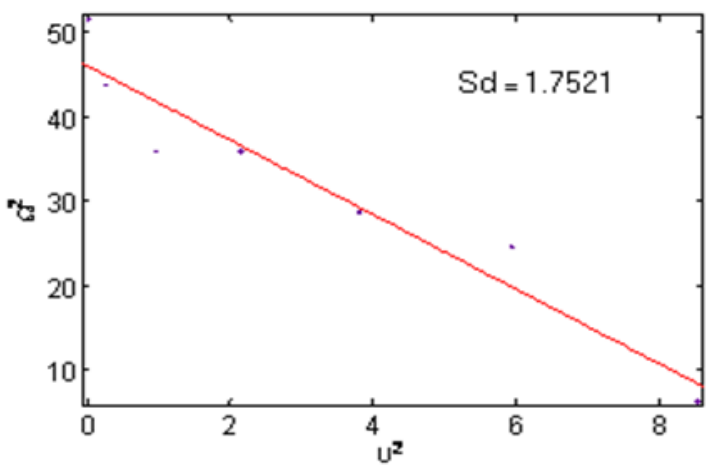

Fig.(15): Experimental plot of $\Omega_{1}{ }^{2}$ against $U^{2}$ for p-p

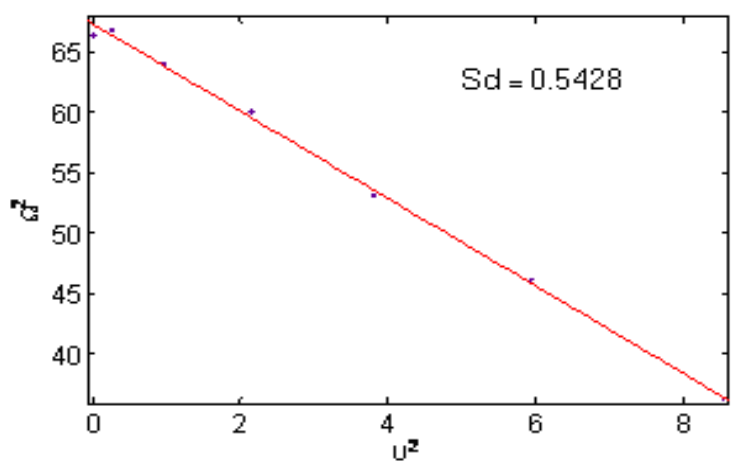

Fig.(16): Experimental plot of $\Omega_{1}^{2}$ against $U^{2}$ for c-p

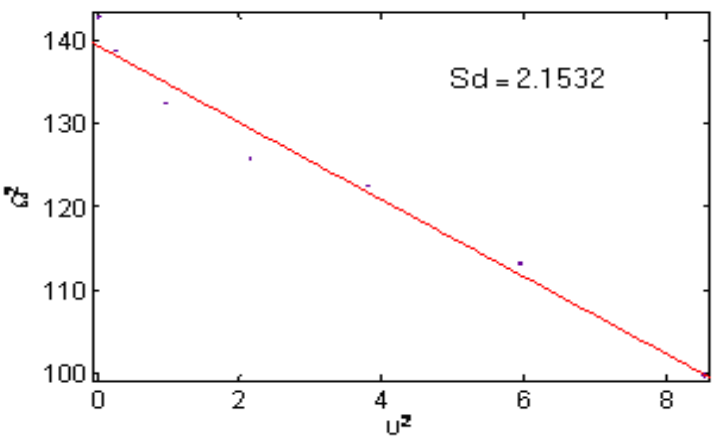

Fig.(17): Experimental plot of $\Omega_{1}^{2}$ against $U^{2}$ for c-c

Table(5): Equations of the fitted lines of figs. (15to 17)

\begin{tabular}{|l|l|l|l|l|l|}
\hline $\begin{array}{l}\text { Figure } \\
\text { no. }\end{array}$ & B.cs & $\begin{array}{l}\text { Equation of } \\
\text { fitted line }\end{array}$ & P1 & P2 & Sd \\
\hline 15 & p-p & $\begin{array}{l}\Omega^{2}=46.05- \\
4.406 \mathrm{U}^{2}\end{array}$ & 4.406 & 46.05 & 1.7521 \\
\hline 16 & c-p & $\begin{array}{l}\Omega^{2}=67.29 \\
3.604 \mathrm{U}^{2}\end{array}$ & 3.604 & 67.29 & 0.5428 \\
\hline 17 & c-c & $\begin{array}{l}\Omega^{2}=139.3- \\
4.63 \mathrm{U}^{2}\end{array}$ & 4.63 & 139.3 & 2.1532 \\
\hline
\end{tabular}

The estimated dimensionless critical velocities were calculated from Eq..(18) .Finally, from Eq.(*) the critical velocities were calculated.
For comparison purpose, the results of the estimated and theoretical critical velocities of the two models and the associated errors are given in Table (6).

Table (6): Estimated and theoretical critical velocities

\begin{tabular}{|l|l|l|l|}
\hline \multirow{2}{*}{$\begin{array}{l}\text { Boundary } \\
\text { conditions }\end{array}$} & \multicolumn{2}{|l|}{ Critical velocity(m/s) } & \multirow{2}{*}{ Error\% } \\
\cline { 2 - 3 } & Estimated & Theoretical & \\
\hline p-p & 18.2238 & 17.7091 & 2.9 \\
\hline c-p & 24.3573 & 25.3664 & -3.95 \\
\hline c-c & 30.9194 & 35.4182 & -12.4 \\
\hline
\end{tabular}

Tables (6) indicate that there is generally some errors between the theoretical and the estimated values of critical velocities .These are due to experimental errors and theoretical assumptions.

Also, Table (6) show that for pinned-pinned and clampedpinned cases, the estimated critical velocities are much closed to the theoretical values since the errors are small. However for clamped-clamped case, the errors are relatively high. This can be attributed to the fact that the critical velocities of clamped-clamped pipe are higher than those of other boundary conditions. This will decrease the slope of the line plotted in $U^{2}-\Omega^{2}$ plane . The small slope means that, the variation in the fluid velocity leads to a small variation in the natural frequencies. This small variation is difficult to detect experimentally unless wider range of velocities is considered .Thus, for accurate estimation the flow rate must be increased for clamped-clamped case.

Finally, the results of the experimental work give a reasonable support for some of interesting theoretical results and phenomena such as; the values of the natural frequencies, critical velocities and the effect of the fluid velocities on the natural frequencies .

\section{CONCLUSIONS}

From the discussions of the theoretical and experimental results of vibration, stability and parametric instability for pipes conveying fluid, the main conclusions will be presented.

1. The general solution for the vibration equation of conservative pipes conveying fluid is derived in this paper .From this solution the frequency equations for pinned-pinned, clamped-pinned and clamped-clamped pipes conveying fluid are obtained semi-analytically in terms of the pipe parameters.

2. In all types of conservative pipes, the natural frequencies decrease with increasing of fluid velocity

3. Increasing the pressure decreases the critical velocities of buckling for all types of conservative pipes.

4. The concept of stability boundary is used as an approximate approach for investigating the sequence of stability for conservative pipes conveying fluid.. This approach provides a simple and effective graphical method for analyzing stability at a wide range of fluid velocities .The validity of this approach is examined by comparing its main results with the available published data. The results show good agreements.

5. A new experimental approach for estimating buckling critical velocities from measuring several natural frequencies at relatively small flow rates is presented. The results show good agreement between the estimated and theoretical critical velocities in case of pinnedpinned and clamped-pinned pipes .However for clampedclamped pipes the accurate estimation requires higher 
flow rates .This method can serve using a relatively low pump discharge and simple fluid circuit instead of high discharge pumps which demand complication in the fluid circuits.

\section{REFERENCES}

[1] Paidousiss M.P." The Canonical Problem of the FluidConveying Pipe and Radiation of the Knowledge Gained to other Dynamics Problems Across Applied Mechanics." J. Sound Vib. Vol.310, 462-492, 2008.R. Caves, Multinational Enterprise and Economic Analysis, Cambridge University Press, Cambridge, 1982. (book style)

[2] Paidousiss, M.P. "Dynamics of flexible slender cylinders in axial flow. Part 1theory. "Journal of Fluid Mechanics, Vol.26, 717-736.1966..

[3] Paidoussis M. P. ," Fluid-Structure Interactions: Slender Structures and Axial Flow", 1. New York: Academic Press;1998..

[4] G.W.Housner'Bending vibration of a pipeline containing flowing fluid", Journal of Applied Mechanics, 1952.

[5] F.L.Noirdson "Vibration of cylindrical tube containing flowing fluid", Kingliga tekniska hogskolans handlingar, Vol.73, 1953.
[6] R.H.Long "Experimental and theoretical study of transverse vibration of a tube containing flowing fluid", Journal of Applied Mechanics, 1955.

[7] R.W.Gregory and Paidoussis "Unstable oscillation of turbular cantilevers conveying fluid", Proceeding of the Royal Society (London) Vol.293, 1966.

[8] O. Doare' and E. Langre "Local and global instability of fluid conveying pipes on elastic foundation", Journal of Fluids and Structures (2002).

[9] Al-Rajihy A.A, "Out-of-plane vibration of an intermediately supported curved tube conveying fluid",M.Sc Thesis, University of Baghdad,1990.

[10] S.R.Chary,C.K.Rao and R.N.Iyengar "Vibration of fluid conveying pipe on Winkler foundation" ,proceeding of the 8th national convention of Aerospace enginners on Aeroelastically,IIT Kharagpur ,India,1993.

[11] Zhang L.and Huang W., 2000. "Nonlinear Dynamical Modeling of Fluid -Structure Interaction of FluidConveying Pipes." J. of Hydrodynamics, Vol.15 (1), 116-128, 2000.

[12] Sundraraian C., "On the Stability of Divergence-Type Elastic Systems", Devel. Theoret. Appl. Mech. Washington. D.C.,475-481,1974. 